Revista PSICOLOGIA, 2015, Vol. 29 (1), 59-67

\title{
Adaptação de uma versão portuguesa da Escala de Delinquência Auto- Relatada Adaptada para adolescentes
}

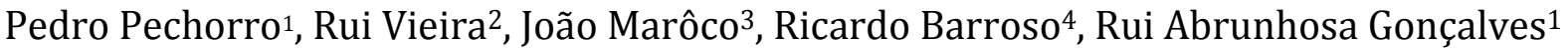 \\ ${ }^{1}$ Escola de Psicologia da Universidade do Minho (EP-UM) \\ ${ }^{2}$ Faculdade de Medicina da Universidade de Lisboa (FM-UL) \\ ${ }^{3}$ ISPA - Instituto Universitário (ISPA-IU) \\ ${ }^{4}$ Universidade de Trás os Montes e Alto Douro (UTAD)
}

\begin{abstract}
Resumo: A presente investigação teve como objetivo adaptar uma versão portuguesa da Escala de Delinquência Auto-Relatada Adaptada (ASRDS), instrumento que avalia comportamentos delituosos e antissociais em adolescentes. Recorrendo a 760 participantes adolescentes subdivididos em amostra forense $(n=250)$ e amostra escolar $(n=510)$ foram demonstradas propriedades psicométricas que na generalidade justificam a sua utilização na população adolescente portuguesa, nomeadamente a nível de validade fatorial, consistência interna, validade divergente, validade convergente, validade concorrente, e validade retrospetiva.
\end{abstract}

Palavras-chave: Avaliação; Delinquência juvenil; Validação; ASRDS.

Adaptation of a Portuguese version of the Adapted Self-Report Delinquency Scale for adolescents: The aim of the present study was to adapt a Portuguese version of the Adapted Self-Report Delinquency Scale (ASRDS), a measure that assesses self-reported delinquent and antisocial behaviors in youths. With a total of 760 youths subdivided in a forensic sample $(n=250)$ and a community sample $(n=510)$ we were able to demonstrate psychometric properties that justify its use with the Portuguese adolescent population in terms of factorial validity, internal consistency, divergent validity, convergent validity, concurrent validity, and retrospective validity.

Keywords: Assessment; Juvenile delinquency; Validation; ASRDS.

As questões de concetualização, definição e medição são fundamentais para o estudo da delinquência juvenil, inclusive nas vertentes mais específicas da sua epidemiologia e da sua etiologia. A maioria dos estudos sobre delinquência anteriores a 1960 eram principalmente baseados em dados oficiais recolhidos pelas autoridades policiais. Com o advento do estudo pioneiro de Short e Nye (1957) foi possível perceber que uma grande maioria dos jovens cometia algum tipo de ato delituoso e que a maioria deste tipo de comportamentos não eram detetados. Tal constatação levou ao desencadear de uma alteração substancial na forma de estudar a delinquência juvenil, que se tem mantido até à atualidade graças à riqueza de dados que proporciona e que dificilmente poderiam ser obtidos de outra forma.

Os questionários de autorresposta formam a base de muito do que é atualmente o conhecimento sobre delinquência juvenil, apesar de alguns autores defenderem ainda que os dados oficiais recolhidos pelas autoridades policiais são mais precisos e válidos (Elliott \& Ageton, 1980; Elliott, Huizinga \& Menard, 1989). Face ao panorama científico atual torna-se imperioso estudar as propriedades psicométricas dos mais difundidos destes instrumentos e, quando possível, adaptá-los à realidade de cada país. Tal é particularmente premente em países como Portugal, em que o tópico da delinquência juvenil tem sido tradicionalmente pouco estudado e em que os dados estatísticos oficiais fornecidos relativos à delinquência juvenil tendem a ser escassos e pouco detalhados.

A Escala de Delinquência Auto-Relatada Adaptada (Adapted Self-Report Delinquency Scale - ASRDS; Carroll, Durkin, Houghton, \& Hattie, 1996) é uma adaptação do instrumento originalmente concebido por Mak (1993), que mede o envolvimento dos adolescentes em atividades ilegais e antissociais. As pontuações obtidas através do somatório dos itens desta escala têm sido utilizadas como um índice de atividade criminal, inclusive para obtenção de valores de incidência e de prevalência.

Carroll et al. (1996) utilizaram duas amostras, de 230 e de 260 adolescentes respectivamente, no

1 Dados de contacto para correspondência: Pedro Pechorro, Escola de Psicologia, Campus de Gualtar, 4710-057 Braga. Portugal. Email: ppechorro@gmail.com. Investigação financiada pela Fundação para a Ciência e a Tecnologia (FCT) com co-financiamento do Fundo Social Europeu POPH/FSE (Bolsa SFRH/BPD/86666/2012). 
processo de adaptação da escala. Os 38 itens da escala foram submetidos a processos de análise fatorial por máxima verosimilhança, tendo sido demonstrado que todos apresentaram saturações superiores a .40. Foram identificados sete fatores, nomeadamente: Roubo e furto (Theft and burglary), Crimes rodoviários (Motor vehicle offenses), Crimes relacionados com drogas e álcool (Drug-related offenses), Agressão (Assault), Vandalismo (Vandalism), Crimes em contexto escolar (School-related offenses) e Perturbação da ordem pública (Public disorder). A consistência interna avaliada pelo alfa de Cronbach de cada um dos fatores identificados variou entre .67 e .91. 0 ajustamento do modelo obtido através de análise fatorial confirmatória foi considerado adequado, $\chi^{2}=1360.3 ; d f=644 ; p<.001 ; \chi^{2} / d f=2.11$; TLI > .09; AGFI > .85. Foi possível também demonstrar que a ASRDS tem validade discriminante ao distinguir entre grupo delinquente e grupos não-delinquentes (Carroll et al., 1996). A investigação empírica já efectuada quanto às propriedades psicométricas da escala tem demonstrado que estas são satisfatórias, apesar de serem necessários procedimentos psicométricos adicionais (e.g., validade convergente e divergente; validade concorrente).

Em Portugal existe a enorme necessidade de proceder à adaptação e validação de instrumentos psicométricos relativos ao campo da delinquência juvenil dada a atual escassez de medidas disponíveis. 0 objetivo do presente artigo consiste em traduzir e adaptar uma versão portuguesa da ASRDS de forma a colmatar a específica falta de medidas de autorresposta que meçam comportamentos delituosos em jovens. Nesse sentido foram colocadas as seguintes hipóteses, nomeadamente que a ASRDS: a) apresenta uma estrutura fatorial com sete dimensões; b) obtém resultados satisfatórios em termos de consistência interna; c) demonstra possuir validade convergente com medidas de traços psicopáticos e de conduta antissocial, além de validade divergente com uma medida de autoestima; d) demonstra validade concorrente com o diagnóstico de perturbação do comportamento, além de validade retrospetiva com as variáveis Idade em que cometeu o primeiro crime e Idade em que teve o primeiro problema com a lei.

\section{MÉTODO}

\section{Participantes}

A amostra ficou constituída por 760 participantes $(M=15.92$ anos; $D P=1.48$ anos), sendo que desse total 250 participantes $(M=15.81$ anos; $D P=1.32$ anos) eram provenientes dos Centros Educativos do Ministério da Justiça e constituíram a amostra forense, e 510 participantes $(M=15.95$ anos; $D P=1.55$ anos) eram provenientes de estabelecimentos públicos de ensino da grande Lisboa e constituíram a amostra escolar. De salientar que o leque etário para participação dos jovens na investigação foi previamente fixado entre os 12 anos e os 20 anos dado ser esse o intervalo etário em que os jovens são passíveis de intervenções ao abrigo da Lei Tutelar-Educativa no sistema judicial português.

No Quadro 1 podem observar-se dados relativos ao sexo, frequência e percentagem de participantes segundo a proveniência.

Quadro 1. Sexo, Número e Percentagem de Participantes por Centros Educativos e Escolas.

\begin{tabular}{|c|c|c|c|c|c|c|}
\hline & $\begin{array}{c}n \text { sexo } \\
\text { masculino }\end{array}$ & $\begin{array}{c}\% \text { sexo } \\
\text { masculino }\end{array}$ & $\begin{array}{c}n \text { sexo } \\
\text { feminino }\end{array}$ & $\begin{array}{c}\% \text { sexo } \\
\text { feminino }\end{array}$ & $N$ Total & \% Total \\
\hline Forense & 221 & 88.4 & 29 & 11.6 & 250 & 32.9 \\
\hline CE B & 56 & 91.8 & 5 & 8.2 & 61 & 8.0 \\
\hline CE NP & 56 & 70.0 & 24 & 30.0 & 80 & 10.5 \\
\hline CE PAO & 32 & 100 & 0 & 0 & 32 & 4.2 \\
\hline CE O & 35 & 100 & 0 & 0 & 35 & 4.6 \\
\hline CE SA & 29 & 100 & 0 & 0 & 29 & 3.8 \\
\hline CE M & 13 & 100 & 0 & 0 & 13 & 1.7 \\
\hline Escolar & 322 & 63.1 & 188 & 36.9 & 510 & 67.1 \\
\hline Escola A & 177 & 61.7 & 110 & 38.3 & 287 & 37.8 \\
\hline Escola MC & 69 & 82.1 & 15 & 17.9 & 84 & 11.1 \\
\hline Escola AA & 45 & 67.2 & 22 & 32.8 & 67 & 8.8 \\
\hline Escola AN & 31 & 43.1 & 41 & 56.9 & 72 & 9.5 \\
\hline Total & 543 & 71.4 & 217 & 28.6 & 760 & 100 \\
\hline
\end{tabular}

Nota. $N$ = número de participantes; \% = percentagem; CE = Centro Educativo; CE B = CE da Belavista; CE NP = CE Navarro de Paiva; CE $P A O=C E$ Padre António de Oliveira; CE $O=C E$ dos Olivais; CE SA = CE Santo António; CE M = CE do Mondego; Escola A = Escola da Amora; Escola $M C=$ Escola do Monte da Caparica; Escola AA = Escola Anselmo de Andrade; Escola AN = Escola Azevedo Neves.

A caracterização sociodemográfica demonstrou que a amostra forense continha menos participantes do sexo feminino, $\chi^{2}=5.484, p \leq .001$, menos participantes de cor branca, $\chi^{2}=38.776, p \leq$ .001 , menos participantes de proveniência urbana, $\chi^{2}=18.580, p \leq .001$, menos anos de escolaridade completos, $F=1194.506, p \leq .001$, mais progenitores com baixo nível socioeconómico, $U=33514, p \leq$ .001 , e mais progenitores divorciados ou falecidos, $\chi^{2}=127.898, p \leq .001$. Não foram encontradas 
diferenças estatisticamente significativas entre a amostra forense e a amostra escolar relativamente à idade dos participantes e à nacionalidade portuguesa.

\section{Instrumentos}

A Escala de Delinquência Auto-Relatada Adaptada (Adapted Self-Report Delinquency Scale - ASRDS; Carroll et al., 1996; Carroll, Houghton, Durkin, \& Hattie, 2009) é uma medida de autorresposta adaptada constituída por 38 itens que mede o envolvimento dos adolescentes em atividades ilegais e antissociais. A ASRDS no presente estudo foi cotada somando os itens ordinais tipo Likert de 3 pontos (Nunca $=0$, Algumas vezes $=1$, Frequentemente $=2$ ) de forma a simplificar as opções de resposta, embora seja possível utilizar versões com opções de resposta de 2 a 7 pontos. Pontuações mais altas indicam maior envolvimento em atividade criminal. As pontuações obtidas na ASRDS podem ser utilizadas como um índice de atividade criminal, inclusive para obtenção de valores de incidência e de prevalência. A ASRDS foi escolhida para a presente investigação dado que em Portugal praticamente não existem escalas validadas que tenham por objetivo medir comportamentos delituosos em jovens.

O Dispositivo de Despiste de Processo Antissocial (Antisocial Process Screening Device - APSD; Frick \& Hare, 2001; Muñoz \& Frick, 2007) é uma medida psicométrica projetada para avaliar traços psicopáticos em crianças e adolescentes. O APSD foi modelado a partir da Psychopathy Checklist - Revised (PCL-R; Hare, 2003) com a colaboração do próprio Hare, tendo por base amostra clínicas e comunitárias. Na presente investigação utilizou-se a versão de autorresposta adaptada a Portugal por Pechorro, Vieira e Vieira (2012) com itens ordinais tipo Likert de três pontos (Nunca $=0$, Algumas vezes $=1$, Frequentemente $=2$ ). Pontuações mais altas indicam níveis de traços psicopáticos mais altos. 0 APSD foi utilizado na presente investigação para efetuar a validade convergente dado que é conhecida a predisposição para os comportamentos delituosos dos indivíduos com pontuações altas em traços psicopáticos (Pechorro, 2011).

A Escala Taxonómica para Crianças e Adolescentes (Child and Adolescent Taxon Scale - CATS; Harris, Rice \& Quinsey, 1994) é uma escala de ordenação actuarial desenvolvida a partir de oito variáveis relacionadas com conduta antissocial e agressiva na infância e adolescência que permitem discriminar os indivíduos pertencentes à categoria antissocial dos não pertencentes. Pontuações mais altas indicam uma maior probabilidade de pertencer à categoria antissocial. Na presente investigação utilizou-se a versão adaptada a Portugal da CATS por Pechorro (2011) para efetuar a validade convergente dado ser uma medida de conduta antissocial que tem apresentado boas propriedades métricas (Quinsey, Harris, Rice, \& Cormier, 2006).

A Escala de Auto-Estima de Rosenberg (Rosenberg Self-Esteem Scale - RSES; Rosenberg, 1989) é uma medida breve de autorresposta que avalia a autoestima em adolescentes e adultos. A RSES pode ser cotada simplesmente somando os dez itens ordinais tipo Likert de 4 pontos (Discordo fortemente $=0$, Discordo $=1$, Concordo $=2$, Concordo fortemente $=3$ ), após se ter efetuado a reversão dos itens apropriados (nomeadamente os itens 2, 5, 6, 8 e 9). Pontuações mais altas indicam níveis de autoestima mais altos. Na presente investigação utilizou-se a versão adaptada a Portugal por Pechorro, Marôco, Poiares e Vieira (2011) para efetuar a validade divergente dado que é uma escala unidimensional que tem apresentado boas propriedades psicométricas em estudos internacionais e não apresenta sobreposição de constructos com medidas de comportamentos delituosos e antissociais (Pechorro, 2011).

Adicionalmente foi construído um questionário para descrever as caraterísticas sociodemográficas dos participantes. Este questionário incluiu questões como a idade dos participantes, a sua nacionalidade, grupo étnico, o sexo, a proveniência rural versus urbana, os anos de escolaridade completados, o nível socioeconómico dos pais e o estado civil dos pais. 0 primeiro autor deste artigo efetuou o diagnóstico de Perturbação do Comportamento de acordo com os critérios do DSM-IV-TR (American Psychiatric Association, 2002). Para analisar o nível socioeconómico utilizou-se a metodologia empregue por Simões (1994).

\section{Procedimentos}

Como princípio do processo de validação obtivemos autorização de Annemaree Carroll para utilizar a ASRDS. Para proceder à tradução dos instrumentos seguiram-se guidelines estabelecidas internacionalmente (Hambleton, Merenda, \& Spielberger, 2005). Contou-se com a colaboração de duas tradutoras independentes bilingues licenciadas em Português-Inglês e professoras do ensino secundário. Uma tradutora fez a tradução para português, tendo a outra feito a respectiva retroversão para inglês, que foi então comparada com o instrumento original. Concluída esta fase de tradução-retroversão foi realizado um estudo pré-teste para analisar a qualidade da tradução, detetar problemas e aperfeiçoar a linguagem de forma a torná-la mais facilmente entendível pelos jovens.

No processo de pré-teste teve-se em conta a realidade local, tal como recomendado por Mak 
(1993), o que levou a que fossem eliminados/fundidos três itens considerados redundantes ou culturalmente descontextualizados. Da escala original com 38 itens, o item 4 ("Roubaste uma bicicleta ou peças de uma bicicleta?") e o item 5 ("Roubaste coisas como, por exemplo, peças de um carro ou mota?") da dimensão Roubo e furto foram reformulados dando origem ao item "Roubaste coisas de outras pessoas (exemplo: telemóvel)?"; o item 9 ("Deste umas voltas com um carro roubado?") e o item 10 ("Roubaste e conduziste o carro roubado?") da dimensão Crimes rodoviários deram origem ao item "Guiaste um carro ou mota roubados?"; o item 33 ("Enganaste alguém pelo telefone?") e o item 35 ("Fizeste telefonemas abusadores?") da dimensão Perturbação da ordem pública deram origem ao item "Fizeste telefonemas a ameaçar ou insultar alguém?". Chegou-se assim à versão final da escala com 35 itens (ver Anexo 1).

Foi colocado um termo de consentimento informado no início de cada questionário, em que era dado conhecimento do carácter voluntário, anónimo e confidencial da participação no estudo. A recolha dos questionários em meio forense decorreu individualmente após se ter obtido autorização por parte da Direcção-Geral de Reinserção e Serviços Prisionais (DGRSP), Ministério da Justiça. Foram feitas aplicações em todos os Centros Educativos existentes na altura a nível nacional. A taxa de participação foi de cerca de $90 \%$. Os motivos para a não-participação incluíram a recusa em participar, a impossibilidade de participar devido ao não entendimento da língua portuguesa e a impossibilidade de participar devido a questões de segurança. Todos os questionários dos jovens que participaram foram considerados válidos.

A recolha dos questionários em meio escolar decorreu após se ter obtido autorização por parte da Direcção-Geral de Educação (DGE), Ministério da Educação. Foram aleatoriamente seleccionadas doze escolas básicas/secundárias da região da grande Lisboa, das quais quatro concordaram em participar. Os motivos da não participação incluíram ausência sistemática de resposta ao pedido de colaboração efetuado pelo investigador, alegadas questões relativas à organização interna das escolas que impossibilitaram a colaboração, além de recusa em colaborar devido ao conteúdo forense do questionário. As escolas que aceitaram participar solicitaram que a participação de cada aluno fosse previamente autorizada através de um termo de consentimento assinado pelo encarregado de educação. No final, foram excluídos cerca de $13 \%$ dos participantes devido a estarem fora do intervalo etário estabelecido ou a motivos como terem entregado questionários não preenchidos, incompletos ou ilegíveis.

Os dados foram inseridos e analisados utilizando o SPSS v. 22 (IBM SPSS, 2013) e posteriormente o EQS 6.2 (Bentler \& Wu, 2008). Após a inserção dos dados ter sido feita foram aleatoriamente selecionados $10 \%$ dos questionários inseridos, de forma a avaliar a qualidade de inserção dos mesmos. A qualidade foi considerada boa dado que praticamente não foram detetados erros de inserção. No tratamento de dados estatísticos propriamente dito recorreu-se a uma variedade de técnicas estatísticas, incluindo estatísticas descritivas, análise fatorial, análise de consistência interna por alfa de Cronbach e correlações paramétricas e não-paramétricas.

A estrutura fatorial da versão Portuguesa da ASRDS foi avaliada com Análise Fatorial Confirmatória (AFC) efetuada no software EQS com métodos robustos de estimação. Os índices de ajustamento calculados incluíram: Qui-quadrado de Satorra-Bentler/graus de liberdade, CFI (Comparative Fit Index), IFI (Incremental Fit Index), RMSEA (Root Mean Square Error of Approximation). Um qui-quadrado/graus de liberdade $\leq 5$ é considerado adequado, se $\leq 2$ é considerado bom e se $=1$ é considerado muito bom (Marôco, 2014; West, Taylor, \& Wu, 2012). Valores CFI $\geq .90$ e RMSEA $\leq .10$ indicam ajustamento adequado, enquanto que CFI $\geq .95$ e RMSEA $\leq .06$ indicam um ajustamento bom (Byrne, 2006). Um valor de IFI $\geq .90$ é considerado aceitável, sendo que se IFI $\geq .95$ é considerado bom. Em caso de necessidade seriam utilizados Índices de Modificação para melhorar o ajustamento.

A AFC foi efetuada diretamente nos itens da ASRDS e somente valores com saturação $\geq .40$ foram considerados. Optou-se pela utilização de uma matriz de correlações policóricas com métodos de estimação robustos nos itens ordinais dado que proporcionam melhores resultados (Byrne, 2006). Correlações Pearson foram utilizadas para analisar as associações entre variáveis escalares, enquanto as correlações bisseriais por ponto foram utilizadas para analisar a relação entre variáveis nominais dicotómicas e variáveis escalares (Leech, Barrett, \& Morgan, 2015). Em termos de magnitude de correlações, consideraram-se correlações fracas as correlações entre 0 e .20, correlações moderadas entre .20 e .50 , e correlações fortes acima de .50 .

\section{RESULTADOS}

$\mathrm{Na}$ fase inicial do tratamento de dados procurou-se confirmar a estrutura fatorial obtida utilizando o programa informático EQS 6.2 (Bentler, 2008). Encontraram evidências através de AFC que apoiaram a estrutura original de 7 fatores, $\mathrm{S}-\mathrm{B} \chi^{2}=1747.56, p \leq .001 ; \chi^{2} / d f=4.69$; IFI = .98; CFI = .98; RMSEA =.06 (.05- .06), e o ajustamento geral foi considerado bom. No Quadro 2 podem-se observar as saturações dos itens, que se mantiveram sempre acima de.40. 
Pechorro, Vieira, Marôco, Barroso e Gonçalves

Quadro 2. Cargas fatoriais para o modelo robusto da ASRDS.

\begin{tabular}{|c|c|c|c|c|c|c|c|}
\hline & Fator 1 & Fator 2 & Fator 3 & Fator 4 & Fator 5 & Fator 6 & Fator 7 \\
\hline Item 1 & .90 & & & & & & \\
\hline Item 2 & .76 & & & & & & \\
\hline Item 3 & .41 & & & & & & \\
\hline Item 4 & .90 & & & & & & \\
\hline Item 5 & .83 & & & & & & \\
\hline Item 6 & & .76 & & & & & \\
\hline Item 7 & & .76 & & & & & \\
\hline Item 8 & & .94 & & & & & \\
\hline Item 9 & & .58 & & & & & \\
\hline Item 10 & & .83 & & & & & \\
\hline Item 11 & & .95 & & & & & \\
\hline Item 12 & & & .62 & & & & \\
\hline Item 13 & & & .63 & & & & \\
\hline Item 14 & & & .83 & & & & \\
\hline Item 15 & & & .51 & & & & \\
\hline Item 16 & & & .78 & & & & \\
\hline Item 17 & & & .54 & & & & \\
\hline Item 18 & & & & .81 & & & \\
\hline Item 19 & & & & .87 & & & \\
\hline Item 20 & & & & .64 & & & \\
\hline Item 21 & & & & .82 & & & \\
\hline Item 22 & & & & & .61 & & \\
\hline Item 23 & & & & & .70 & & \\
\hline Item 24 & & & & & .84 & & \\
\hline Item 25 & & & & & .49 & & \\
\hline Item 26 & & & & & .44 & & \\
\hline Item 27 & & & & & .47 & & \\
\hline Item 28 & & & & & & .83 & \\
\hline Item 29 & & & & & & .68 & \\
\hline Item 30 & & & & & & .81 & \\
\hline Item 31 & & & & & & & .46 \\
\hline Item 32 & & & & & & & .42 \\
\hline Item 33 & & & & & & & .66 \\
\hline Item 34 & & & & & & & .67 \\
\hline Item 35 & & & & & & & .57 \\
\hline
\end{tabular}

Nota. Fator 1 = Roubo e furto; Fator 2 = Crimes rodoviários; Fator 3 = Crimes relacionados com drogas e álcool; Fator 4 = Agressão;

Fator 5 = Vandalismo; Fator 6 = Crimes em contexto escolar; Fator 7 = Perturbação da ordem pública

No Quadro 3 apresentamos as correlações entre a ASRDS e as suas dimensões.

Quadro 3. Matriz de correlações Pearson da ASRDS e suas dimensões.

\begin{tabular}{|c|c|c|c|c|c|c|c|c|}
\hline & ASRDS & Fator 1 & Fator 2 & Fator 3 & Fator 4 & Fator 5 & Fator 6 & Fator 7 \\
\hline ASRDS & 1 & & & & & & & \\
\hline Fator 1 & $.91^{* * *}$ & 1 & & & & & & \\
\hline Fator 2 & $.81^{* * *}$ & $.66^{* * *}$ & 1 & & & & & \\
\hline Fator 3 & $.82^{* * *}$ & $.68^{* * *}$ & $.61^{* * *}$ & 1 & & & & \\
\hline Fator 4 & $.90^{* * *}$ & $.84^{* * *}$ & $.73^{* * *}$ & $.65^{* * *}$ & 1 & & & \\
\hline Fator 5 & $.77^{* * *}$ & $.67 * * *$ & $.52^{* * *}$ & $.56^{* * *}$ & $.66^{* * *}$ & 1 & & \\
\hline Fator 6 & $.84^{* * *}$ & $.77^{* * *}$ & $.58^{* * *}$ & $.60^{* * *}$ & $.74^{* * *}$ & $.63^{* * *}$ & 1 & \\
\hline Fator 7 & $.82^{* * *}$ & $.73^{* * *}$ & $.52^{* * *}$ & $.67^{* * *}$ & $.66^{* * *}$ & $.60 * * *$ & $.70^{* * *}$ & 1 \\
\hline
\end{tabular}

Nota. Fator 1 = Roubo e furto; Fator 2 = Crimes rodoviários; Fator 3 = Crimes relacionados com drogas e álcool; Fator 4 = Agressão; Fator 5 = Vandalismo; Fator 6 = Crimes em contexto escolar; Fator 7 = Perturbação da ordem pública

*** Estatisticamente significativo ao nível .001

Seguidamente calculou-se o alfa de Cronbach, as médias das correlações inter-itens e o leque de correlações item-total corrigidas (ver Quadro 4). 
Quadro 4. Alfas de Cronbach, médias das correlações inter-itens e leque de correlações item-total corrigidas para a ASRDS e suas dimensões.

\begin{tabular}{lccc}
\hline & $\alpha$ Cronbach & MCII & LCITC \\
\hline ASRDS total & .96 & .38 & $.32-.80$ \\
1 Roubo e furto & .87 & .57 & $.41-.84$ \\
2 Crimes rodoviários & .92 & .66 & $.58-.88$ \\
3 Crimes com drogas e álcool & .82 & .43 & $.45-.69$ \\
4 Agressão & .86 & .61 & $.56-.79$ \\
5 Vandalismo & .76 & .35 & $.39-.66$ \\
6 Crimes em contexto escolar & .82 & .60 & $.62-.74$ \\
7 Perturbação da ordem pública & .69 & .30 & $.34-.57$ \\
\hline
\end{tabular}

Nota. $\alpha$ Cronbach = Alfa de Cronbach; MCII = Correlação média inter-item; LCITC = Leque de correlações item-total corrigidas.

Na validade convergente obtiveram-se correlações estatisticamente significativas com o APSD, $r=$ .66; $p \leq .01$, e com a CATS, $r=.46 ; p \leq .01$. Em termos de validade divergente com a Escala de Auto-estima de Rosenberg (RSES) foi obtida uma correlação negativa estatisticamente significativa, $r=-.13 ; p \leq .01$. A validade concorrente com o diagnóstico de Perturbação de Comportamento do DSM-IV-TR (American Psychiatric Association, 2002) na amostra forense revelou uma correlação positiva estatisticamente significativa, $r_{p b}=.40 ; p \leq .01$. A validade retrospetiva para a amostra total com a variável Idade em que cometeu o primeiro crime e com a variável Idade em que teve o primeiro problema com a lei revelou correlações negativas estatisticamente significativas tanto na primeira variável, $r=-.44 ; p \leq .01$, como na segunda variável, $r=-.43 ; p \leq .01$.

No Quadro abaixo são apresentadas as estatísticas descritivas da ASRDS por amostras e por sexo dos participantes (ver Quadro 5).

Quadro 5. Estatísticas descritivas da pontuação total da ASRDS por amostras e sexo.

\begin{tabular}{|c|c|c|c|c|}
\hline & \multicolumn{2}{|c|}{ Amostra Forense } & \multicolumn{2}{|c|}{ Amostra Escolar } \\
\hline & Masculino & Feminino & Masculino & Feminino \\
\hline $\mathrm{n}$ & 221 & 29 & 322 & 188 \\
\hline$M$ & 30.77 & 20.28 & 9.44 & 6.45 \\
\hline$D P$ & 13.59 & 10.03 & 9.09 & 5.13 \\
\hline Mínimo & 4 & 3 & 0 & 0 \\
\hline Máximo & 63 & 51 & 52 & 27 \\
\hline Assimetria & .311 & .918 & 2.110 & 1.301 \\
\hline EP Assimetria & .164 & .434 & .136 & .177 \\
\hline Curtose & -.719 & 1.92 & 5.807 & 2.131 \\
\hline EP Curtose & .326 & .845 & .271 & .353 \\
\hline
\end{tabular}

Nota. $n=$ número de participantes; $M=$ Média; $D P=$ Desvio-padrão; EP Assimetria = Erro-padrão da Assimetria; EP Curtose = Erropadrão da Curtose.

\section{DISCUSSÃO}

O ponto de partida para a presente investigação foi a adaptação para a realidade linguística e cultural portuguesa da ASRDS, escala que mede o envolvimento dos adolescentes em atividades ilegais e antissociais. Nesse sentido, foram colocadas algumas hipóteses em termos das propriedades psicométricas esperadas na adaptação nacional da ASRDS, nomeadamente no que diz respeito a estrutura fatorial, consistência interna, validade convergente e divergente, validade concorrente e validade retrospetiva.

Como passo inicial procedeu-se a CFA da ASRDS. Foi possível encontrar a estrutura original com sete fatores da escala (Carroll et al., 1996) sendo que todos os itens saturaram adequadamente dado terem atingido valores iguais ou superiores a .40 (Nunnally \& Bernstein, 1994). Recordamos que Carroll et al. (1996) adotaram uma postura mais conservadora ao reterem apenas itens com saturação superior a .40. Relativamente às correlações entre a ASRDS e as suas dimensões, estas foram sempre fortes e estatisticamente significativas conforme esperado. Desta forma, foi possível confirmar a primeira hipótese colocada.

Seguidamente calculou-se a consistência interna por alfa de Cronbach, as médias das correlações inter-itens e o leque de correlações item-total corrigidas. Os resultados dos alfas foram bons por serem maioritariamente iguais ou superiores a .70 na amostra total e nas dimensões (Cortina, 1993), sendo mesmo superiores aos que Carroll et al. (1996) obtiveram. A única exceção foi a dimensão Perturbação da ordem pública, que obteve um alfa de .69, muito perto do valor mínimo recomendado de .70. Os 
resultados das médias das correlações inter-itens revelaram alguns problemas dado que as dimensões Roubo e furto, Crimes rodoviários, Agressão, e Crimes em contexto escolar obtiveram valores acima do intervalo recomendado de .15 a .50 (Clark \& Watson, 1995), o que revela excessiva homogeneidade dos itens que as constituem. 0 leque de correlações item-total corrigidas foi considerado bom para a ASRDS e suas dimensões dado que atingiu sempre a correlação mínima recomendada de .20 (Nunnally \& Bernstein, 1994). Confirmámos, portanto, a segunda hipótese colocada.

A validade convergente demonstrou uma correlação moderada alta e significativa com o APSD e uma correlação moderada significativa com a CATS, evidenciando assim sobreposição com os constructos de traços psicopáticos e de conduta antissocial (Kline, 2000). Tal era expectável dado que têm vindo a ser demonstradas na literatura as associações entre estes constructos e a frequência de atividades delituosas e antissociais (e.g., Pechorro, Maroco, Gonçalves, Nunes, \& Jesus, 2014). A validade divergente com a RSES obteve uma correlação baixa e negativa, demonstrando a fraca sobreposição com o constructo de autoestima que era esperada. A validade concorrente com o diagnóstico de Perturbação de Comportamento do DSM-IV revelou uma correlação moderada e estatisticamente significativa. A validade retrospectiva com a variável Idade em que cometeu o primeiro crime e com a variável Idade em que teve o primeiro problema com a lei demonstrou correlações moderadas negativas e significativas (Domino \& Domino, 2006; Nunnally \& Bernstein, 1994), evidenciando a relação existente entre a idade de início na atividade criminal e a frequência de crimes cometidos (e.g., Patterson, Forgatch, Yoerger, \& Stoolmiller, 1998). Estes aspetos da adaptação da ASRDS são especialmente importantes dado que não foram analisados por Carroll et al. (1996). Conclui-se, portanto, que também a terceira hipótese e a quarta hipótese foram confirmadas.

De uma forma geral, consideramos que foi possível evidenciar propriedades psicométricas suficientemente adequadas da ASRDS na nossa amostra. Em termos de limitações do nosso estudo, vale a pena referir que teria sido interessante comparar as respostas dos jovens detidos em Centro Educativo com os dados oficiais do Ministério da Justiça sobre esses jovens, caso esses dados tivessem estado disponíveis e não houvesse obrigação de anonimato. Futuramente será necessário continuar o processo de validação efetuando outros procedimentos (e.g., validação cruzada). Tal como referiram Nunnally e Bernstein (1994) a validação de um instrumento é um processo sempre inacabado. Os investigadores forenses portugueses têm agora à sua disposição um instrumento breve de autorresposta fiável que podem utilizar para avaliar a delinquência juvenil no contexto nacional.

\section{Referências}

American Psychiatric Association (2002). Manual de diagnóstico e estatística das perturbações mentais (4⿻ ed., revisão de texto). Lisboa: Climepsi Editores.

Bentler, P. (2008). EQS 6 structural equations program manual. Encino, CA: Multivariate Software, Inc.

Byrne, B. (2006). Structural equation modeling with EQS: Basic concepts, applications, and programming. Mahwah, NJ: Lawrence Erlbaum Associates.

Carroll, A., Durkin, K., Houghton, S., \& Hattie, J. (1996). An adaptation of Mak's self-report delinquency scale for western Australian adolescents. Australian Journal of Psychology, 48, 1-7.

Carroll, A., Houghton, S., Durkin, K., \& Hattie, J. (2009). Adolescent reputations and risk: Developmental trajectories to delinquency. New York, NY: Springer.

Clark, L., \& Watson, D. (1995). Constructing validity: Basic issues in objective scale development. Psychological Assessment, 7, 309-319.

Corcoran, K., \& Fischer, J. (2000), Measures for clinical practice: A sourcebook (3 ${ }^{\text {rd }}$ ed., Vol. 1). New York: The Free Press.

Cortina, J. (1993). What is coefficient alpha? An examination of theory and applications. Journal of Applied Psychology, 78, 98-104.

Domino, G., \& Domino, M. (2006). Psychological testing: An introduction (2 ${ }^{\text {nd }}$ ed.). New York: Cambridge University Press.

Elliott, D., \& Ageton, S. (1980). Reconciling race and class differences in self-reported and official estimates of delinquency. American Sociological Review, 45, 95-110.

Elliott, D., Huizinga, D., \& Menard, S. (1989). Multiple problem youth: Delinquency, substance abuse and mental health problems. New York: Springer-Verlag.

Frick, P., \& Hare, R. (2001). The Antisocial Process Screening Device: Technical manual. Toronto, Canada: Multi-Health System.

Hambleton, R., Merenda, P., \& Spielberger, C. (Eds.). (2005). Adapting educational and psychological tests for cross-cultural assessment. Mahwah, NJ: LEA Publishers.

Hare, R. (2003). The Hare Psychopathy Checklist-Revised: Technical manual (2 ${ }^{\text {nd }}$ ed.). Toronto, Canada: Multi-Health Systems. 
Harris, G., Rice, M., \& Quinsey, V. (1994). Psychopathy as a taxon: Evidence that psychopaths are a discrete class. Journal of Consulting and Clinical Psychology, 62, 387-397.

IBM SPSS. (2013). IBM SPSS Statistics Base 22. Chicago, IL: SPSS Inc.

Leech, N., Barrett. K., \& Morgan, G. (2015). SPSS for intermediate statistics: Use and interpretation (5 ${ }^{\text {th }}$ ed.). New York: Routledge.

Kline, P. (2000). The handbook of psychological testing. London: Routledge.

Mak, A. (1993). A self-report delinquency scale for Australian Adolescents. Australian Journal of Psychology, 45, 75-79.

Marôco, J. (2011). Análise estatística com o SPSS Statistics. Pêro Pinheiro: ReportNumber.

Marôco, J. (2014). Análise de equações estruturais: Fundamentos teóricos, software e aplicações. Pêro Pinheiro: ReportNumber.

Muñoz, L., \& Frick, P. (2007). The reliability, stability, and predictive utility of the self-report version of the Antisocial Process Screening Device. Scandinavian Journal of Psychology, 48, 299-312.

Nunnaly, J., \& Bernstein, I. (1994). Psychometric theory. New York: McGraw-Hill.

Patterson, F., Forgatch, M., Yoerger, K., \& Stoolmiller, M. (1998) Variables that initiate and maintain an early-onset trajectory for juvenile offending. Development and Psychopathology, 10, 531-547.

Pechorro, P. (2011). Delinquência juvenil: Estudo de algumas variáveis psicológicas e relacionais com ênfase nos traços psicopáticos (Dissertação de doutoramento não-publicada). Faculdade de Medicina da Universidade de Lisboa, Lisboa.

Pechorro, P., Vieira, R., \& Vieira, D. (2012). Adaptação e validação preliminar duma versão portuguesa do Dispositivo de Despiste de Processo Anti-social. Laboratório de Psicologia, 10, 97-110.

Pechorro, P., Marôco, J., Poiares, C., \& Vieira, R. (2011). Validação da Escala de Auto-Estima de Rosenberg com adolescentes portugueses em contexto forense e escolar. Arquivos de Medicina, 25, 174-179.

Pechorro, P., Maroco, J., Gonçalves, R., Nunes, C., \& Jesus, S. (2014). Psychopathic traits and age of crime onset in male juvenile delinquents. European Journal of Criminology, 11, 288-302.

Piquero, A., MacIntosh, R., \& Hickman, M. (2002). The validity of a self-reported delinquency scale. Sociological Methods \& Research, 30, 492-529.

Quinsey, V., Harris, V., Rice, M., \& Cormier, C. (2006). Violent offenders: Appraising and managing risk (2 ${ }^{\text {nd }}$ ed.). Washington, DC: American Psychological Association.

Rosenberg, M. (1979). Conceiving the self. New York: Basic Books.

Rosenberg, M. (1989). Society and the adolescent self-image (Revised edition). Middletown: Wesleyan University Press.

Simões, M. (1994). Investigação no âmbito da aferição nacional ao Teste das Matrizes Progressivas Coloridas de Raven (MPCR) (Tese de doutoramento não publicada). Faculdade de Psicologia e Ciências da Educação da Universidade de Coimbra, Coimbra.

Short, J., Jr., \& Nye, F. (1957). Reported behavior as a criterion of deviant behavior. Social Problems, 5 , 207-213.

West, S., Taylor, A., \& Wu, W. (2012). Model fit and model selection in structural equation modeling. In R. Hoyle (Ed.), Handbook of structural equation modeling (pp. 209-231). New York: The Guilford Press.

Historial do artigo

Recebido 29/04/2014

Aceite 12/04/2015

Publicado $06 / 2015$

Apoio à publicação: Fundação para a Ciência e a Tecnologia (Ministério da Educação e Ciência, Portugal) - Programa FACC 
Anexo 1. Itens da versão portuguesa da ASRDS.

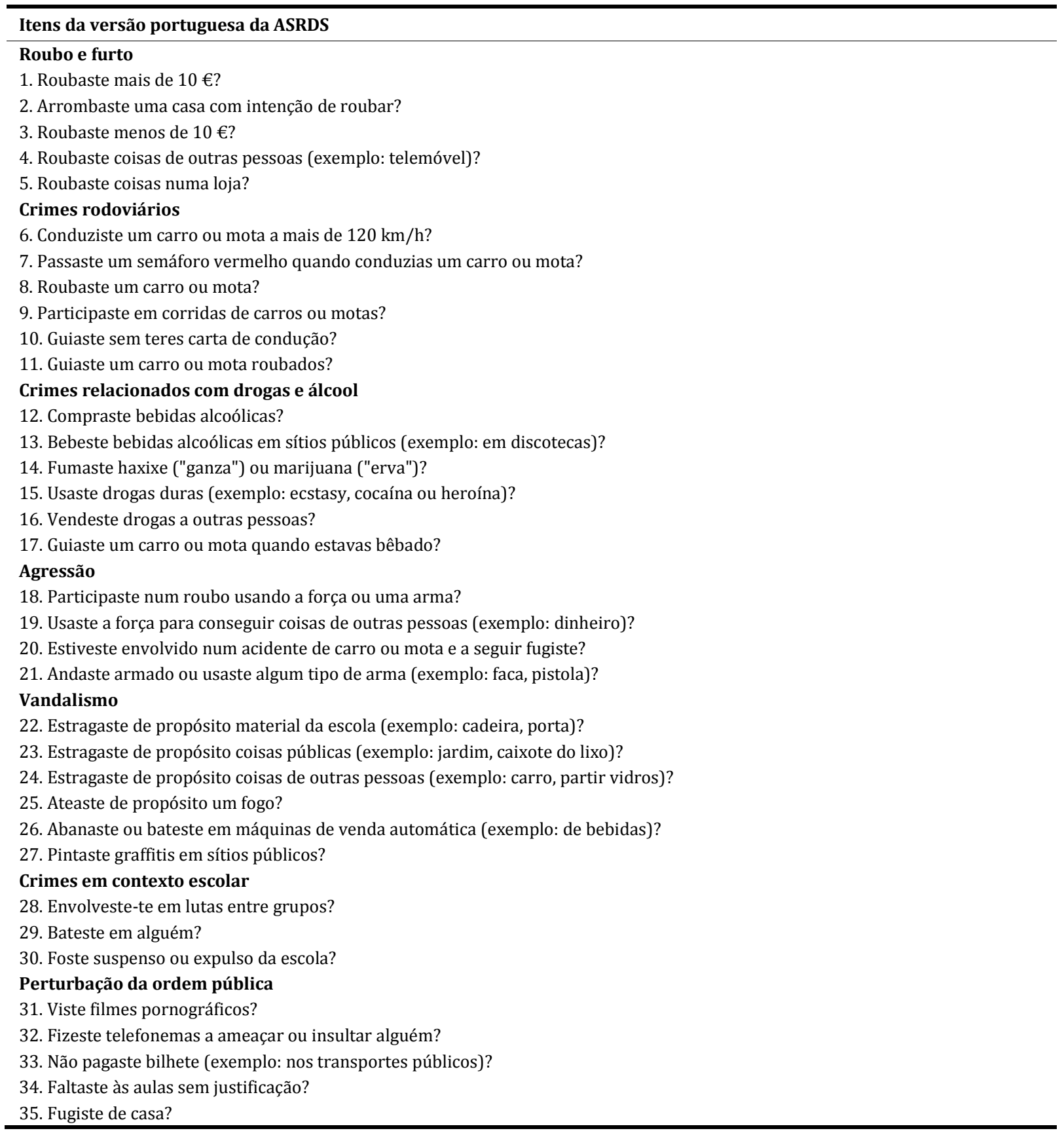

